\title{
ESTENOSIS LARINGOTRAQUEAL Revisión de casos en el Centro Médico Imbanaco de Cali
}

\author{
Antonio José Reyes Solarte, $M D^{1}$ \\ ${ }^{1}$ Otorrinolaringólogo, Universidad del Valle \\ Centro Médico Imbanaco de Cali
}

\begin{abstract}
Resumen
El manejo de la estenosis laringotraqueal continúa siendo un problema difícil para el otorrinolaringólogo por la multiplicidad de hallazgos en los pacientes, su manejo complejo y la falta de un tratamiento único. En el Centro Médico Imbanaco de Cali, Colombia, se realizó un análisis retrospectivo de una serie de pacientes con este diagnóstico tratados entre octubre de 2006 y enero de 2011. Se examinaron los antecedentes, síntomas y signos, tratamientos, enfermedades asociadas, resultados del tratamiento y seguimiento en el tiempo. Se identificaronun total de 44 adultos, con edad promedio de 41,4 años; 24 (55\%) eran mujeres y 20 hombres. El promedio de seguimiento fue 19 meses; 28 $(63,6 \%)$ pacientes mejoraron con el empleo de tratamiento endoscópico y láser de CO2. Los mejores resultados se obtuvieron en el Grupo I (100\%) y en el Grupo II (83.3\%). La cirugía endoscópica láser, es un tratamiento válido para lesiones delgadas, no complicadas. El uso de técnicas de resección y reconstrucción laringotraqueal es útil en las lesiones complejas y gruesas donde falla el tratamiento endoscópico. No obstante, en casos seleccionados, el tratamiento endoscópico se debe considerar inicialmente. Otras ventajas del tratamiento endoscópico son; pocas complicaciones, baja morbilidad, tiempo quirúrgico y de hospitalización cortos.
\end{abstract}

Palabras clave: Estenosis traqueal, estenosis laríngea, enfermedades laríngeas, cirugía endoscópica, resultados.

\section{LARYNGO-TRACHEAL STENOSIS. Case studies in Centro Médico Imbanaco in Cali, Colombia}

\begin{abstract}
The management of laryngotracheal stenosis remains a challenging problem for the otolaryngologist. The complexity of the various preoperative situations implies that no single treatment modality can solve the problem. I performed a retrospective analysis of our experience in Centro Médico Imbanaco in Cali, Colombia, with a series of patients with this condition between October 2006 and January 2011. The study involved retrospective review of the records of patients with laryngotracheal stenosis. Symptoms, antecedents, treatment, associate pathology, outcomes and follow up were examined. A total of 44 adults were identified, the mean age was 41,4 years, 24 were female (55\%) and 20 were male. The mean follow-up time was 19 months. Twenty eight (63.6\%) of these cases have been controlled effectively with endoscopy laser techniques. Best results were seen in Group I (100\%) and Group II (83.3\%). Endoscopic laser surgery is a valid approach for thinner, uncomplicated lesions; patients with thicker, complex lesions in whom endoscopic treatment fails are best managed with laryngotracheal resection a reconstruction, but endoscopic treatment may be considered before
\end{abstract}

* e-mail: ajreyes@imbanaco.com.co 
tracheal resection in select cases. Endoscopic treatment is associated with few complications, low morbidity, a short operative time, and a short length of hospitalization.

Key words: Tracheal stenosis, laryngeal stenosis, laryngeal diseases, endoscopic surgical procedure, outcomes

\title{
ESTENOSE LARINGOTRAQUEAL Revisão de casos no Centro Médico Imbanaco de Cali
}

\begin{abstract}
Resumo
O tratamento da estenose laringotraqueal continua sendo um problema difícil para o otorrinolaringologista pela multiplicidade de descobertas nos pacientes, seu tratamento complexo e a falta de um tratamento único. No Centro Médico Imbanaco de Cali, Colômbia, realizou-se uma análise retrospectiva de uma série de pacientes com este diagnóstico, tratados entre outubro de 2006 e janeiro de 2011. Foram examinados os antecedentes, sintomas e signos, tratamentos, doenças associadas, resultados do tratamento e acompanhamento no tempo. Foram identificados um total de 44 adultos, com idade média de 41,4 anos; 24 (55\%) eram mulheres e 20 homens. A média de acompanhamento foi 19 meses; 28 (63,6\%) pacientes melhoraram com o emprego de tratamento endoscópico e laser de CO2. Os melhores resultados foram obtidos no Grupo I (100\%) e no Grupo II (83.3\%). A cirurgia endoscópica laser, é um tratamento válido para lesões finas, não complicadas; o uso de técnicas de resecção e reconstrução laringotraqueal são úteis nas lesões complexas e grossas onde falha o tratamento endoscópico; não obstante, em casos selecionados, o tratamento endoscópico deve ser considerado inicialmente. Outras vantagens do tratamento endoscópico são poucas complicações, baixa morbilidade, tempo cirúrgico e de hospitalização curtos.
\end{abstract}

Palavras chave: Estenose traqueal; estenose laríngea; doenças laríngeas; cirurgia endoscópica; resultados.

\section{Introducción}

Las enfermedades que padecemos son el reflejo de los cambios en los estilos de vida y los progresos en la atención médica. Hace unos años la mayoría de los casos de estenosis laringotraqueal eran secundarios a procesos infecciosos, tales como difteria, sífilis e ingestión de cáusticos. Actualmente la intubación laringotraqueal y el trauma en accidentes de tránsito son las principales causas de estenosis (1). La mayoría de estas lesiones comprometen más de un sitio en la laringe y la tráquea. En las estenosis laringotraqueales se deben tener en cuenta la etiología, el sitio y la severidad. El sitio de la estenosis es el principal factor al seleccionar el tipo de tratamiento, mientras que la severidad de la estenosis orienta sobre la probabilidad de éxito (2). La complejidad en las diferentes situaciones prequirúrgicas implica que no hay una modalidad simple de tratamiento que resuelva todos los problemas. Existe una variedad de manejos que van desde tratamientos con láser con o sin dilatación o colocación de stent, reconstrucción laringotraqueal con injertos de cartílago costal, resección cricotraqueal parcial para los grados más severos de estenosis y cirugía extendida en casos de estenosis glótico subglótica combinada (3). En este artículo se reporta la experiencia en el manejo de la estenosis laringotraqueal en el Centro Médico Imbanaco de Cali, entre los años 2006 y 2011.

\section{Materiales y métodos}

El diseño del estudio es de tipo descriptivo retrospectivo, en una serie de casos operados en el Centro Médico Imbanaco comprendidos entre los años 2006 y 2011. Se diseñó una base de datos en Excel y se analizaron los resultados con la ayuda del programa SPSS versión 18.0 (4). El análisis estadístico descriptivo se hizo con base en distribuciones porcentuales para variables categóricas y cálculo de medidas de 
tendencia central y variabilidad para variables continuas. Para comparar los resultados finales, según los diferentes grados de estenosis, se aplicó la prueba de probabilidad exacta de Fisher (5). Se estableció a priori un nivel de significación estadística $\alpha=0,05$.

\section{Pacientes}

Entre octubre de 2006 y enero de 2011, se atendieron 59 pacientes con diagnóstico de estenosis laringotraqueal en el Centro Médico Imbanaco de Cali (CMI) por parte de los otorrinolaringólogos Adriana O'Byrne y Antonio José Reyes. Se realizó tratamiento endoscópico a $15(25,4 \%)$ pacientes en edad pediátrica y a $44(74,6 \%)$ adultos ( $>17$ años). Este reporte se refiere a los pacientes adultos tratados durante ese tiempo. Los datos fueron extractados de las historias clínicas del CMI y de los registros de consulta externa de los médicos mencionados. El seguimiento final se realizó de manera presencial en consultorio por parte de los médicos tratantes y en algunos pacientes se realizó entrevista telefónica. El seguimiento osciló entre tres meses y cuatro años con un promedio de seguimiento de 19 meses. La recolección de datos se centró en la etiología, tipo y sitio de la estenosis, antecedentes de importancia, tipo y número de tratamientos realizados, enfermedades asociadas, éxito y causas de falla. Para la estadificación de los pacientes se utilizaron las clasificaciones de Bogdasarian y Olson en las personas con estenosis glótica y la clasificación de McCaffrey en los enfermos con estenosis laringotraqueal (Tablas 1 y 2).

Tabla 1. Clasificación de Bogdasarian y Olson para Estenosis glótica

\begin{tabular}{|c|l|}
\hline Grado & \multicolumn{1}{|c|}{ Localización de la estenosis } \\
\hline I & Sinequia interaritenoidea limitada a los procesos vocales sin comprometer la comisura posterior \\
\hline II & Cicatriz que ocupa todo el plano interaritenoideo, sin fijar la articulación cricoaritenoidea \\
\hline III & Cicatriz de la comisura posterior con fijación de una articulación cricoaritenoidea \\
\hline IV & Cicatriz de la comisura posterior con fijación de ambas articulaciones cricoaritenoideas \\
\hline
\end{tabular}

Tabla 2. Clasificación de McCaffrey para Estenosis laringotraqueal en adultos

\begin{tabular}{|c|l|}
\hline Grado & \multicolumn{1}{|c|}{ Localización de la estenosis } \\
\hline I & Subglotis o tráquea $<1 \mathrm{~cm}$ de largo (membrana) \\
\hline II & Subglotis confinada al anillo cricoideo, o tráquea cervical $>1 \mathrm{~cm}$ largo \\
\hline III & Subglotis, envuelve el anillo cricoideo y se extiende a la tráquea superior, \\
\hline IV & Cualquier estenosis con compromiso glótico (estrechamiento o parálisis) \\
\hline
\end{tabular}

\section{Técnica quirúrgica}

Se empleó técnica endoscópica cerrada, como tratamiento primario o secundario. Esta técnica utiliza el láser de $\mathrm{CO}_{2}$, diferentes sistemas de dilatación y aplicación tópica de mitomicina $\mathrm{C}$, la cual ha mostrado resultados en la inhibición de la neo-angiogénesis y de la proliferación de fibroblastos (6). Se realizaron bajo anestesia general con ventilación espontánea o con el uso de ventilación jet cuando lo permitían las condiciones del paciente o a través de la cánula de traqueotomía cuando estaba presente; se utilizó laringoscopio de suspensión (Dedo, Kleinsasser o de comisura anterior) el cual se avanza a través de los pliegues vocales para identificar la estenosis subglótica o traqueal. El láser de $\mathrm{CO}_{2}$, en superpulso $3.5 \mathrm{~W}$, spot de $270 \mu$, con una distancia focal de $400 \mathrm{~mm}$ y a 0.10 segundos de repetición, para minimizar la difusión de calor a los tejidos vecinos. En los casos de estenosis concéntrica se utilizó la técnica de Shapshay (7). empleando incisiones radiales en los cuatro puntos cardinales y dejando puentes de mucosa entre las incisiones con el láser que facilitan el proceso de reepitelización. La dilatación se realizó con dilatadores 
de Hegar, y otros dilatadores que se diseñaron para este fin, se fabricaron en acero inoxidable, de punta redondeada, de diferentes calibres y un mango en ángulo de 45 grados los cuales permiten una buena visualización. Las dilataciones se realizaron en forma delicada, evitando rasgar la mucosa, y se aplicó mitomicina $\mathrm{C}$ en forma tópica con una torunda de algodón a una concentración de $2 \mathrm{mg} / \mathrm{ml}$ durante dos o tres minutos.

\section{Criterios de éxito}

Se consideró éxito quirúrgico cuando el paciente presentó resolución de la sintomatología (disnea, estridor), decanulación en los pacientes traqueostomizados y voz funcional cuando el paciente había tenido compromiso de la glotis. Cuando no se cumplen los criterios anteriores se considera una falla.

\section{Resultados}

En este estudio se reportan los hallazgos en 44 pacientes, 20 hombres y 24 mujeres, con edades entre 18 y 86 años (promedio 39,8 $\pm 15,1$ años); 4 (9,1\%) pacientes presentaban lesiones a nivel glótico y 40 (90,9\%) tenían estenosis laringotraqueal.

Las lesiones glóticas se estadificaron de acuerdo con la clasificación de Bogdasarian (8) y en las lesiones subglóticas y traqueales se utilizó la clasificación de McCaffrey (9). Dos (50\%) pacientes estudiados con estenosis glótica posterior se clasificaron como grado I, 1 (25\%) grado II, 1 (25\%) se clasificó como grado III y ninguno como grado IV. La mayor parte de los pacientes del estudio presentaron hallazgos de estenosis laringotraqueal y se clasificaron así: 6 (15\%) pacientes grado I, 24 (60\%) grado II, 4 (10\%) grado III, y 6 (15\%) grado IV.

La consistencia de la lesión se discriminó como blanda 17 (38,6\%) y dura 27 (61,4\%). La longitud de la lesión se clasificó como menor de un centímetro, 28 $(63,6 \%)$, entre uno y dos centímetros, $12(27 \%)$ y mayor de $2 \mathrm{~cm}, 4$ (9,1\%); 37 pacientes tenían antecedente de intubación $(84,1 \%)$, con un rango entre 1 y 30 días (promedio 12,1 \pm 5,3 días); 33 (75\%) pacientes llegaron con traqueotomía, 4 (9,1\%) tenían antecedentes de procedimientos endoscópicos realizados en otra institución, y a $7(15,9 \%)$ se les habían realizado procedimientos abiertos.
Las enfermedades asociadas con el problema de vía aérea fueron las siguientes: $11(25 \%)$ pacientes con intoxicación por organofosforados, $4(9,1 \%)$ accidente de moto; trauma craneoencefálico, septicemia, escleroma laríngeo y herida por arma de fuego en 3 $(6,8 \%)$ respectivamente; cirugía cardíaca e infección pulmonar en 2 (4,5\%). Se encontró un caso de policondritis recurrente $(2,3 \%)$, y otras enfermedades en $8(20,5 \%)$ pacientes (Tabla 3$)$.

Los resultados fueron los siguientes:

Se intervinieron seis pacientes con estenosis subglótica clasificados como grado I (McCaffrey) a los cuales se le realizó un promedio de $1,5 \pm 0,6$ procedimientos (rango 1-3) con éxito de 100\%; 24 pacientes grado II se les realizó un promedio de 2,3 $\pm 1,2$ procedimientos (rango 1-7) con éxito de 83.3\%; 4 pacientes grado III con $4 \pm 1,2$ procedimientos (rango $2-7$ ) sin éxito, y 6 grado IV con $3 \pm 1,4$ procedimientos (rango $1-4)$ con éxito de $33,3 \%$ (Tabla 4).

No hubo fallas en el grupo I; las fallas del tratamiento en el grupo II fueron en dos pacientes no decanulados, una señora de 86 años con historia de carcinoma gástrico e infección pulmonar, y un hombre de 37 años con intoxicación con órgano fosforados; una paciente de 60 años con estridor laríngeo y secuelas de accidente cerebro vascular, y un paciente de 38 años al que se le realizó cirugía abierta con historia de accidente de tránsito severo. Estos cuatro pacientes tenían lesiones que comprometían la tráquea superior entre 1 y $2 \mathrm{~cm}$ de largo. Las fallas en el tratamiento en el grupo III fueron así: dos pacientes a quienes se les realizó cirugía abierta, un hombre de 23 años, otro de 32 años con lesiones secundarias a accidente de tránsito, un paciente de 20 años con disnea, con historia de intoxicación por organofosforados y otro de 19 años que tenía una fístula traqueocutánea. En el grupo IV tres pacientes no se decanularon, uno de 66 años con antecedente de cirugía cardíaca, otro de 59 años con diagnóstico de septicemia y otro de 21 con intoxicación por organofosforados. El cuarto paciente de 40 años con diagnóstico de escleroma laríngeo presenta disfonía.

El porcentaje de éxito en los grupos I (100\%) y II $(83,3 \%)$ fue mayor que en los grupos $\operatorname{III}(0,0 \%)$ y IV $(33,3 \%)$. Estas diferencias fueron estadísticamente significantes a nivel $\alpha=0,05$ (Tabla 4). 
Tabla 3. Hallazgos generales

\begin{tabular}{|c|c|}
\hline $\begin{array}{l}\text { EDAD (años) } \mathbf{n} \\
\text { Media } \pm \text { D. estándar } \\
\text { Rango } 18-86 \\
\text { Mediana } \\
\text { Rango intercuartil }\end{array}$ & $\begin{array}{c}44 \\
39,8 \pm 15,1\end{array}$ \\
\hline $\begin{array}{l}\text { SEXO n (\%) } \\
\text { Masculino } \\
\text { Femenino }\end{array}$ & $\begin{array}{l}44(100.0) \\
20(45,0) \\
24(55,0)\end{array}$ \\
\hline $\begin{array}{l}\text { DIAGNÓSTICO } \mathbf{n}(\mathbf{\%}) \\
\text { Estenosis glótica } \\
\text { Estenosis subglótica }\end{array}$ & $\begin{array}{c}44(100.0) \\
4(9,1) \\
40(90,9)\end{array}$ \\
\hline $\begin{array}{l}\text { EXTENSIÓN LESIÓN, Estenosis SUBGLOTICA n (\%) } \\
\text { Grado I } \\
\text { Grado II } \\
\text { Grado III } \\
\text { Grado IV }\end{array}$ & $\begin{array}{c}40(100.0) \\
6(15,0) \\
24(60,0) \\
4(10,0) \\
6(15,0)\end{array}$ \\
\hline $\begin{array}{l}\text { EXTENSIÓN LESIÓN, Estenosis glótica n (\%) } \\
\text { Grado I } \\
\text { Grado II } \\
\text { Grado III } \\
\text { Grado IV }\end{array}$ & $\begin{array}{l}4(100.0) \\
2(50,0) \\
1(25,0) \\
1(25,0) \\
0(0,0)\end{array}$ \\
\hline $\begin{array}{l}\text { CONSISTENCIA } \mathbf{n}(\mathbf{\%}) \\
\text { Blanda } \\
\text { Dura }\end{array}$ & $\begin{array}{l}44(100.0) \\
17(38,6) \\
27(61,4)\end{array}$ \\
\hline $\begin{array}{l}\text { LONGITUD (cm) n (\%) } \\
<1 \\
1-2 \\
>2\end{array}$ & $\begin{array}{c}44(100.0) \\
28(63,6) \\
12(27,3) \\
4(9,1)\end{array}$ \\
\hline $\begin{array}{l}\text { ANTECEDENTE DE INTUBACIÓN n (\%) } \\
\text { Media } \pm \text { D. estándar } \\
\text { Rango }\end{array}$ & $\begin{array}{l}37(84,1) \\
12,1 \pm 5,3 \\
1-30\end{array}$ \\
\hline $\begin{array}{l}\text { ANTECEDENTE DE } \mathbf{n}(\%) \\
\text { TRAQUEOTOMÍA } \\
\text { PROCEDIMIENTOS ENDOSCÓPICOS } \\
\text { PROCEDIMIENTOS ABIERTOS }\end{array}$ & $\begin{array}{l}33(75,0) \\
4(9,1 \%) \\
7(15,9 \%)\end{array}$ \\
\hline
\end{tabular}




\begin{tabular}{|l|c|}
\hline PATOLOGÍA ASOCIADA & $11(25,0 \%)$ \\
Intoxicación por organofosforados & $4(9,1 \%)$ \\
Accidente tránsito (moto) & $3(6,8 \%)$ \\
\hline TCE & $3(6,8 \%)$ \\
Escleroma laríngeo & $3(6,8 \%)$ \\
ACV & $3(6,8 \%)$ \\
Herida arma fuego & \\
Septicemia & $2(4,5 \%)$ \\
Cirugía cardiaca & $2(4,5 \%)$ \\
Infección pulmonar & $9(22,7 \%)$ \\
Otros & 44 \\
\hline SEGUIMIENTO (meses) $\mathbf{n}$ & $19,05 \pm 12,80$ \\
Media \pm D. estándar & $3-48$ \\
Rango & \\
\hline
\end{tabular}

Tabla 4. Resultados pacientes con estenosis subglotica

\begin{tabular}{|c|c|c|c|c|c|c|}
\hline Grado & $\begin{array}{c}\text { No } \\
\text { Pacientes }\end{array}$ & Éxito & \% éxito & $\begin{array}{c}\text { No Promedio } \\
\text { Procedim }\end{array}$ & $\begin{array}{c}\text { Desv. } \\
\text { prom. }\end{array}$ & Rango \\
\hline I & 6 & 6 & $100,0 \%$ & 1,5 & 0,6 & $1-3$ \\
\hline II & 24 & 20 & $83,3 \%$ & 2,29 & 1,2 & $1-7$ \\
\hline III & 4 & 0 & $0,0 \%$ & $4 ` 1,2$ & $2-7$ & \\
\hline IV & 6 & 2 & $33,3 \%$ & 3 & 1,4 & $1-4$ \\
\hline
\end{tabular}

Grado I vs Grado III - Test exacto de Fisher=0,004 Diferencias estadísticamente significantes Grado I vs Grado IV - Test exacto de Fisher $=0,03$ Diferencias estadísticamente significantes Grado II vs Grado III - Test exacto de Fisher $=0,003$ Diferencias estadísticamente significantes Grado II vs Grado IV - Test exacto de Fisher $=0,029$ Diferencias estadísticamente significantes

Los resultados con los pacientes con estenosis glótica fueron los siguientes: dos pacientes grado I (Bogdasarian) a quienes se les realizó un procedimiento a cada uno con $50 \%$ de éxito; un paciente clasificado como grado II se le realizó un procedimiento endoscópico; y un paciente grado III a quien se le realizaron tres procedimientos y no tuvieron buenos resultados. Las fallas se explican así: el paciente grado I con herida por bala en cuello persistió con disfonía moderada; el paciente grado II con herida en cuello por bala persistió con disfonía; el paciente grado III secundario a accidente de tránsito, persistió con cánula de traqueotomía a pesar de procedimientos como aritenoidectomia y cordectomía posterior, por parálisis de pliegues vocales bilateral, debidas a su accidente.

\section{Seguimiento}

El seguimiento final se realizó en forma personal por los médicos mencionados y en algunos pacientes por entrevista telefónica. El seguimiento osciló entre 3 meses y 4 años con un promedio de seguimiento de 19 meses.

\section{Discusión}

El manejo de la estenosis laringotraqueal permanece como un reto para el otorrinolaringólogo. Se debe hacer un enfoque secuencial en el diagnóstico y el tratamiento para obtener los mejores resultados. En el enfoque diagnóstico de las estenosis laringotraquea- 
les se debe tener en cuenta los siguientes aspectos: la etiología, el sitio y la severidad de la estenosis. El sitio de la estenosis es el principal factor al considerar la selección del tipo de tratamiento, mientras que la severidad de la estenosis orienta la probabilidad de éxito en el tratamiento.

Entre las causas de estenosis se encuentran las neoplasias, la infección, el trauma por intubación, la enfermedad por reflujo gastroesofágico (ERGE) y algunas enfermedades inflamatorias e idiopáticas (1). Las neoplasias más comunes de la vía aérea superior son de origen epitelial, de crecimiento lento o rápido, los tumores del tiroides, benignos o malignos; las neoplasias del tejido conectivo de la vía aérea tales como condromas o condrosarcomas, las neoplasias glandulares como carcinomas adenoidoquísticos o carcinomas mucoepidermoides. El tratamiento de la obstrucción por neoplasia de la vía aérea está definido por el tipo y localización del tumor. Las infecciones virales o bacterianas necrotizantes severas de la vía aérea superior pueden acarrear daño y pérdida de la mucosa, lo cual puede dar como resultado estenosis de la vía aérea. Se considera como un factor agravante en los daños producidos por los tubos endotraqueales en la vía aérea que terminan en estenosis.

Entre las infecciones específicas conocidas como causantes de estenosis están la sífilis, el rinoescleroma, la blastomicosis y la tuberculosis. La intubación endotraqueal es una causa común de estenosis laringotraqueal; la duración de la intubación es uno de los principales factores de estenosis, sin embargo, el tipo y tamaño del tubo, la presión del balón, la infección concurrente, el movimiento del tubo, y la reintubación pueden incrementar la incidencia de daño laringotraqueal y la estenosis después de la intubación. Los sitios donde existe mayor riesgo de daño son los sitios de contacto del tubo endotraqueal, estos son la tráquea cervical que está en contacto con el balón del tubo, y el punto donde el tubo descansa en la laringe posterior en el espacio interaritenoideo. Puede pasar inadvertido el trauma cerrado del cuello anterior, con frecuencia asociado conotros traumas. Una fractura laríngea, usualmente con laceración de la mucosa, puede llevar a estenosis tardía debido a que muchos pacientes con trauma severo del cuello están intubados, y estas laceraciones logran permanecer inadvertidas, hasta que el paciente no se logra extubar. La enfermedad por reflujo gastroesofágico (ERGE) ha estado implicada al comienzo y en el progreso de la estenosis de la vía aérea superior. El reflujo a veces es silencioso, hasta que se manifiestan los síntomas de obstrucción progresiva de la vía aérea. Algunas enfermedades inflamatorias afectan la mucosa o los tejidos conectivos de la vía aérea superior dando como resultado una estenosis; entre estas enfermedades están la granulomatosis de Wegener, la policondritis recurrente y LES. Otras enfermedades inflamatorias con compromiso significativo de la mucosa son el penfigoide cicatricial y la colitis ulcerativa que se presenta como estenosis laringotraqueal. En algunas series de estenosis laringotraqueal existe un subgrupo de pacientes a los cuales no se les encontró una causa aparente (10) y fue más prevalente en mujeres las cuales tienen una vía aérea más estrecha. Algunas de estas estenosis se relacionan con ERGE o Wegener, y algunos son secundarios a infección necrotizante de la mucosa después de una infección respiratoria severa (11).

\section{Diagnóstico}

Una historia cuidadosa de los síntomas sugiere con frecuencia la etiología e indica la severidad. Los factores a considerar son la instalación, el progreso, la severidad y los factores agravantes.

Los síntomas incluyen estridor, disnea con el ejercicio, lo cual puede ser de instalación súbita o gradual. Las estenosis laringotraqueales a veces muestran progreso o estabilización. Sin embargo, las estenosis secundarias a condiciones inflamatorias crónicas o reflujo gastroesofágico son más susceptibles de progresar comparadas con las estenosis asociadas con un evento único como un trauma externo o daño post-intubación. En general la severidad de los síntomas de la estenosis laringotraqueal se correlacionan con las dimensiones de la vía aérea. Los pacientes a veces permanecen asintomáticos o con síntomas leves hasta que la luz de la vía aérea alcanza un nivel crítico de $4 \mathrm{~mm}$, donde los síntomas se tornan progresivos. Los síntomas aumentan con el ejercicio, debido a la dependencia no linear de la resistencia de la vía aérea. El estridor durante el reposo es un síntoma tardío de estenosis laringotraqueal severa. Cuando se presentan síntomas de obstrucción de la vía aérea como sibilancias y como la estenosis laringotraqueal no es una entidad común, estos se diagnostican como asma en forma inapropiada. El diagnóstico de asma se descarta con la prueba de función pulmonar que muestran una obstrucción de la vía aérea extra 
torácica fija. La tos de tipo perruno es relativamentefrecuente; la retención de secreciones e irritación de receptores de la vía aérea secundaria a turbulencias da inicio al reflejo de la tos.

Una nasofibrolaringoscopia cuidadosa de la laringe y de la tráquea en el consultorio es el paso más importante en la evaluación de la estenosis glótica y subglótica, para lo cual es necesario aplicar la anestesia local en forma adecuada, ya sea tópica, nebulizada o instilada por vía transtraqueal. Es importante capturar las imágenes en un medio digital o de video para una revisión posterior detallada. Se deben valorar los siguientes aspectos durante el examen, movilidad de los pliegues vocales, cicatrices en la comisura posterior, examen a través de la subglotis, documentación de las medidas de la vía aérea, y examen retrógrado de la vía aérea subglótica en caso de existir una traqueotomía (3).

Otros exámenes son útiles en la evaluación de la estenosis laringotraqueal. La granulomatosis de Wegener se diagnostica midiendo los niveles de anticuerpos anticitoplasma de neutrófilo (ANCA). La policondritis recurrente no tiene prueba de laboratorio, sin embargo, una eritrosedimentación elevada y otros indicadores de inflamación crónica, correlacionan con los hallazgos clínicos. La impedanciometría con Phmetría es útil en aquellos casos que se sospeche ERGE como causante de estenosis laringotraqueal. Hasta ahora no se conoce su especificidad y sensibilidad en esta enfermedad. Los estudios imagenológicos son esenciales para la evaluación de la estenosis laringotraqueal. Una radiografía lateral del cuello puede mostrar reducción del calibre de la vía aérea. Una tomografía con reconstrucción tridimensional es muy útil en el diagnóstico. La reconstrucción coronal es necesaria para determinar el largo y la dimensión de la estenosis. Es de fácil realización y da medidas de la dimensión de la estenosis y su localización con respecto de la glotis. La resonancia magnética nuclear es otra técnica útil. Las pruebas funcionales tienen alguna utilidad para evaluar la resistencia de la vía aérea y determinar la severidad de la estenosis. Sin embargo, la relación no linear entre la resistencia de la vía aérea y estenosis hace que esta técnica sea útil solo para probar los síntomas subjetivos del paciente. La curva flujo-volumen es una medida útil del grado de obstrucción del paciente. Un flujo inspiratorio máximo menor de 2 litros por segundo indica un nivel de obstrucción alto de la vía aérea.

\section{Tratamiento}

El objetivo de la reparación de la estenosis laringotraqueal es restablecer una vía aérea a las dimensiones normales que permanezca estable con los cambios de presión durante el esfuerzo respiratorio. Se han propuesto diferentes clasificaciones de acuerdo con el sitio y el compromiso de los tejidos, siendo estas importantes para definir el tipo de procedimiento a realizar. Las clasificaciones utilizadas en nuestro servicio son las de McCaffrey para estenosis subglótica y la de Bogdasarian para la estenosis glótica (Tablas 1 y 2). La clasificación por grupos de las estenosis es un predictor excelente para determinar la posibilidad de éxito de un tratamiento. Se puede esperar que 90\% de los grupos I y II puedan ser tratadas con éxito, mientras que solo $40 \%$ de los pacientes del grupo IV pudieron ser decanulados (1), hallazgos encontrados en la revisión de los pacientes del CMI. Aun con un tratamiento adecuado, las lesiones estenóticas que comprometen la glotis son menos exitosas en su manejo, que aquellas que comprometen la subglotis y la tráquea no siendo posible algunas veces restaurar la función de la glotis.

Existen tres tipos de técnicas quirúrgicas básicas. La primera son las técnicas endoscópicas que consisten en incisiones radiales múltiples del área de la estenosis con el láser de $\mathrm{CO}_{2}$ seguido por la dilatación con la punta de un broncoscopio. Este procedimiento conserva la mucosa presente en el sitio de la estenosis y minimiza el riesgo de reestenosis, de los segmentos dilatados. La segunda, es la resección segmentaria, que se realiza desde el nivel de la tráquea al cartílago cricoides y se repara por anastomosis primaria. En la estenosis traqueal, la reanastomosis primaria de la tráquea se realiza después de movilizar la tráquea vecina. Se puede hacer una resección segmentaria del cartílago cricoides, si la estenosis no se acerca más de $1.5 \mathrm{~cm}$ de los pliegues vocales. En las estenosis que se extienden más arriba del punto medio del cartílago cricoides, resección y reanastomosis son técnicamente difíciles. La tercera es la expansión por laringotraqueoplastia la cual se hace por la división anterior y posterior del cartílago cricoides y tiroides colocando un injerto de cartílago en la división anterior o posterior o en ambos sitios.

Entre los procedimientos endoscópicos se encuentra que la dilatación es útil para el tratamiento de estenosis delgadas pudiendo las estenosis de menos de $0.5 \mathrm{~cm}$ 
de largo ser tratadas por este método. Esta técnica implica instalar el broncoscopio o los dilatadores dentro de la vía aérea y después pasarlo a través de la estenosis. Para estenosis más grandes, este procedimiento se inicia con incisiones radiales con el láser en 3 ó 5 puntos alrededor de la circunferencia de la estenosis con la técnica descrita por Shapshay (7). Se han descrito buenos resultados con esta técnica en diferentes trabajos publicados en la literatura $(2,12,13,14,15)$. La experiencia en el CMI con este tratamiento muestra óptimos resultados en los pacientes clasificados como grado I y II. La escisión láser de la estenosis subglótica o traqueal tiene limitaciones. La escisión de estenosis de grado alto sacrifica una cantidad significante de epitelio; a pesar de que con esto se incrementan las dimensiones de la vía aérea, la escisión con láser produce una contracción adicional y una reestenosis de la vía aérea.

En los casos en que se haga la escisión de porciones sustanciales de la vía aérea, es necesaria la colocación de un stent (16) para permitir reepitelización de la mucosa resecada y evitar la contracción; esto es útil para estenosis donde no se realiza una resección segmentaria o una expansión. Este puede ser el caso del paciente con riesgo quirúrgico alto, o con la posibilidad de progresión de la estenosis, como es el caso de la estenosis por una neoplasia. Se coloca un stent después de una dilatación o incisión o escisión con láser de una estenosis traqueal, o ambas. Es motivo de controversia el uso de stent de malla metálica. Algunos autores han mostrado buenos resultados con este procedimiento; existe un gran riesgo de progresión de la estenosis y formación de tejido de granulación (16).

La resección segmentaria se utiliza en segmentos de tráquea desde 3 a $5 \mathrm{~cm}$ de largo (17). La técnica de escisión traqueal y reconstrucción se utiliza si el segmento estenótico está confinado a la tráquea por debajo del cartílago cricoides. El sitio de la estenosis está definido por estudio de imágenes y broncoscopia preliminar. Cuando se resecan segmentos mayores de $3 \mathrm{~cm}$ de largo, la liberación de la laringe puede ser necesaria para reducir la tensión de la anastomosis. En algunos casos el nivel de la resección se lleva dentro del cartílago cricoides. Es posible resecar el borde inferior del cartílago cricoides y realizar una anastomosis cricotraqueal. Otra técnica descrita es el avanzamiento de la tráquea sobre el cricoides, formando un cuello alrededor de la tráquea.
La laringotraqueoplastia por expansión comprende la incisión vertical del cartílago laríngeo y de la tráquea con una expansión lateral, con o sin injertos del cricoides. Variaciones de esta técnica incluyen abrir únicamente el cartílago, cricoides anterior y posterior sin injertos, o expandir la vía aérea usando injerto anterior y posterior. El propósito de la laringotraqueoplastia de expansión es incrementar las dimensiones de la vía aérea y producir una vía aérea estable que contrarreste las fuerzas y los cambios de presión relacionados con el ciclo respiratorio (18).

Laringotraqueoplastia con Split cricoideo anterior: la apertura anterior del cricoides es útil para incrementar las dimensiones de la vía aérea subglótica, al liberar las fuerzas de constricción del tejido cicatricial, siendo la apertura anterior simple útil en estenosis leves que envuelvan el cricoides. El cartílago se divide en la línea media y hasta el primer y segundo anillo traqueal. Si esto resulta en expansión de la vía aérea con una fuerza mínima, se puede insertar un stent interno temporal y permitir que la vía aérea cicatrice por fibrosis sobre el stent, obteniendo una expansión limitada.En la mayoría de los casos es necesaria la inserción de un injerto de cartílago anterior, obtenido del cartílago costal, para superar las fuerzas de reestenosis. Si la incisión vertical anterior no permite suficiente expansión de la vía aérea para restaurar las dimensiones necesarias, es necesario un Split posterior. En la laringotraqueoplastia con Split cricoideo anteroposterior, la apertura del cricoides anteroposterior se realiza por división de la porción inferior del cartílago tiroides, el cartílago cricoides, y el primero y el segundo anillos traqueales. El cartílago es retraído para exponer el anillo cricoideo posterior, el cual se divide al liberar el cartílago cricoides obteniendo una mayor expansión que únicamente con la apertura anterior. La integridad estructural de la vía aérea es restaurada con injertos en el anillo anterior y posterior del cricoides que se obtienen del cartílago costal. Esta técnica da una expansión de $4 \mathrm{~mm}$ posteriormente y $5 \mathrm{~mm}$ anteriormente. La expansión se puede lograr desde la glotis a la tráquea (7). La ventaja de esta técnica es la posibilidad de expandir el área interaritenoidea sin comprometer la función de la articulación cricoidea. La desventaja es la incapacidad de expandir la tráquea por debajo del primer o segundo anillo traqueal. La mayoría de las técnicas de laringotraqueoplastia con apertura anterior o posterior requiere del uso de injertos. Se utiliza cartílago costal, cartílago septal, cartílago auricular. En los casos que requieren poca expansión se utiliza 
hueso hioides o cartílago tiroides. Cada uno de ellos tiene sus ventajas. El cartílago costal da un injerto cartilaginoso grande pero requiere la exposición de otro campo quirúrgico. El cartílago septal tiene una cantidad limitada de material. La oreja da una cantidad limitada de cartílago y no tiene la rigidez del septum o costilla. El uso del hueso hioides o el cartílago tiroides tiene la ventaja de poder obtenerse del mismo campo quirúrgico, pero la cantidad de tejido es limitada. Para una cicatrización óptima de la vía aérea reconstruida se requiere estabilizar el cartílago que ha sido dividido; esto se realiza con injertos y fijación interna rígida o semirrígida. Si los injertos no se utilizan, la fijación con stent es necesaria para estabilizar los fragmentos de cartílago durante el proceso de cicatrización. Si la fijación rígida no se puede realizar, aun con injertos, el stent es necesario para dar soporte durante la cicatrización inicial.

\section{Conclusiones}

El manejo de la estenosis laringotraqueal es un reto grande que demanda el concurso de equipos multidisciplinarios con entrenamiento en este campo. Entre los diferentes tipos de tratamiento de la estenosis laringotraqueal que se han descrito, se incluye el uso del láser, las dilataciones endoscópicas repetidas, la colocación de stents, los diferentes tipos de reconstrucción laringotraqueal y la resección segmentaria con anastomosis termino-terminal. La opción de tratamiento ideal debe ser individualizada basada en las características del paciente y las ventajas y desventajas de cada procedimiento.

Los resultados obtenidos en la serie revisada están en concordancia con los resultados obtenidos en otros centros e informados en la literatura.

Los procedimientos endoscópicos tienen excelentes resultados en las estenosis subglóticas clasificadas como Grado I y II.

\section{Referencias bibliográficas}

1. McCaffrey TV. Laryngotracheal stenosis. En: Ossof RH, Shapshay SM, Woodson GE, Netterville JL, editores. The larynx. Philadelphia: Lipppincott Williams \& Wilkins; 2003. p. 244.

2. Monnier P. Subglottic and tracheal stenosis. En: Remacle M, Eckel HE, editores. Surgery of larynx and trachea. Berlin: Sringer-Verlag; 2010. p. 139-40.

3. Rosen C, Simson CB. Glottic an subglottic stenosis: evaluation and surgical planning. Chapter 6.En: Operative techniques in laryngology. Berlin: Sprlinger-Verlag; 2008. p. 37-42.

4. $I B M \otimes S P S S \AA$. Statistics is a comprehensive, easy-to-use set of data and predictive analytics tools for business users, analysts and statistical programmers. Disponible en: http://www-01. $\mathrm{ibm}$.com/software/ lk/analytics/spss/products/statistics/

5. Pertega S, Pita S. Asociación de variables cualitativas: el test exacto de Fisher y el test de McNemar. Cad Aten Primaria. 2004; 11: 304-11.

6. Hardillo J, Vanclooster C, Delacre PR. An investigation of airway wound healing using a novel in vivo model. Laryngoscope. 1984; 111: 1174-82.

7. Shapshay SM, Beamis JF, Hybels RL, Bohigian RK. Endoscopic treatment of subglottic and tracheal stenosis by radial laserincision and dilatation. Ann Otol Rhinol Laryngol. 1987; 96: 661-4.

8. Bogdasarian RS, Olson NR. Posterior glottic laryngeal stenosis. Otolaryngol Head Neck Surg. 1980; 88: 765-72.

9. McCaffrey TV. Classification of laryngotracheal stenosis. Laryngoscope. 1992; 102: 1335-40.

10. Valdéz TA, Shapshay SM. Ideopathic subglottic stenosis revisited. Ann Otol Rhinol Laryngol. 2002; 111: 690-5.

11. Mcllwain JC. A historical overview of the aetiology and treatment of laryngeal stenosis. Arch Otolaryngol. 1989; 246:336-40.

12. Rahbar R, Shapshay S, Healy G. Mitomycin: Effects on laryngeal and tracheal stenosis, benefits, and complications. Ann Otol Rhinol Laryngol. 2001; 110: 1-6.

13. Samaniego G, López A. Estenosis laringotraqueal: estudio retrospectivo. Rev Sanid Mil. 2000; 54: 2009-13.

14. Schweinfurth JM. Endoscopic treatment of severe tracheal stenosis. Ann Otol Rhinol Laryngol. 2006; 115: 30-4.

15. Debnath I, Paniello R, Spector JG. Laryngeal stenosis.En:Fried M, Ferlito A, editores. The larynx. San Diego: Plural Publishing; 2009. p.137-49.

16. Sato Y, Imamura H. Airway stenting. Surg Today. 2005; 35 : 265-70.

17. Grillo HC. Management of idiopathic tracheal stenosis. Chest Surg Clin N Am. 1996; 6: 811-8.

18. George M, Lang F, Pasche P, Monnier P. Surgical management of laringotracheal stenosis in adults. Eur Arch Otolarnygol. 2005; 262: 609-15. 